\title{
The effectiveness of an anti-human IL-6 receptor monoclonal antibody combined with chemotherapy to target colon cancer stem-like cells
}

\author{
JIN YING*, MASAHIKO TSUJII", JUMPEI KONDO, YOSHITO HAYASHI, MOTOHIKO KATO, \\ TOMOFUMI AKASAKA, TAKUTA INOUE, ERI SHIRAISHI, TAHAHIRO INOUE, SATOSHI HIYAMA, \\ YOSHIKI TSUJII, AKIRA MAEKAWA, SHOICHIRO KAWAI, TETSUJI FUJINAGA, MAEKAWA ARAKI, \\ SHINICHIRO SHINZAKI, KENJI WATABE, TSUTOMU NISHIDA, HIDEKI IIJIMA and TETSUO TAKEHARA
}

\author{
Department of Gastroenterology and Hepatology, Osaka University Graduate \\ School of Medicine, Suita, Osaka 565-0871, Japan
}

Received September 30, 2014; Accepted November 19, 2014

DOI: 10.3892/ijo.2015.2851

\begin{abstract}
Recent studies have demonstrated that cancer stem cells (CSCs) can initiate and sustain tumor growth and exhibit resistance to clinical cytotoxic therapies. Therefore, CSCs represent the main target of anticancer therapy. Interleukin-6 (IL-6) promotes cellular proliferation and drug resistance in colorectal cancer, and its serum levels correlate with patient survival. Therefore, IL-6 and its downstream signaling molecule the signal transducer and activator of transcription-3 (STAT3) represent potential molecular targets. In the present study, we investigated the effects of IL-6 and its downstream signaling components on stem cell biology, particularly the chemoresistance of CSCs, to explore potential molecular targets for cancer therapy. The colon cancer cell line WiDr was cultured in serum-free, non-adherent, and three-dimensional spheroid-forming conditions to enrich the stem cell-like population. Spheroid-forming cells slowly proliferated and expressed high levels of Oct-4, Klf4, Bmi-1, Lgr5, IL-6, and Notch 3 compared with adherent cells. Treatment with an antihuman IL-6 receptor monoclonal antibody reduced spheroid formation, stem cell-related gene expression, and 5-fluorouracil (5-FU) resistance. In addition, IL-6 treatment enhanced the levels of p-STAT3 (Tyr705), the expression of Oct-4, Klf4, Lgr5, and Notch 3, and chemoresistance to 5-FU. siRNA targeting Notch 3 suppressed spheroid formation, Oct-4 and Lgr5 expression, and 5-FU chemoresistance, whereas STAT3 inhibition enhanced Oct-4, Klf4, Lgr5, and Notch 3 expression and 5-FU chemoresistance along with reduced spheroid growth. Taken together, these results indicate that IL-6 func-
\end{abstract}

Correspondence to: Professor Tetsuo Takehara, Department of Gastroenterology and Hepatology, Osaka University Graduate School of Medicine, Suita, Osaka 565-0871, Japan

E-mail: takehara@gh.med.osaka-u.ac.jp

*Contributed equally

Key words: IL-6, STAT3, Notch 3, cancer stem cells, colon cancer tions in dichotomous pathways involving Notch 3 induction and STAT3 activation. The former pathway is involved in cancer stem-like cell biology and enhanced chemoresistance, and the latter pathway leads to accelerated proliferation and reduced chemoresistance. Thus, an anti-human IL-6 receptor monoclonal antibody or Notch 3 inhibition may be superior to STAT3 inhibition for CSC-targeting therapies concomitant with anticancer drugs.

\section{Introduction}

Colorectal cancer is the third most common visceral malignancy in the world and remains one of the leading causes of cancer-related deaths, primarily due to resistance to therapy (1). Recently, tumor initiation and metastases have been suggested to be dependent on a small sub-population of tumor cells termed cancer stem cells (CSCs), which exhibit infinite self-renewal potential and the capacity to differentiate into diverse populations (2). The first evidence for the existence of CSCs was observed in the context of acute myeloid leukemia; since then, an increasing number of studies have described CSCs in solid tumors, including breast, lung, liver, brain, melanoma, prostate, ovarian, and colon cancer. CSCs share all of the fundamental traits of stem cells, such as self-renewal by asymmetric division, reduced proliferation and differentiation, and enhanced resistance to apoptosis (3). Therefore, therapies targeting CSCs have been proposed to improve the efficacy of currently available cancer treatments.

One of the characteristics of tumor-derived CSCs is that they can grow in spheroids in vitro when plated in limited numbers under suspension conditions in a serum-free, defined media supplemented with growth factors (4). Spheroidforming assays have been developed as a method to estimate stem-like properties, as reported in studies of neurospheres (5), mammospheres (6) and colonospheres (7). However, little is known about the signaling events that regulate the growth and maintenance of colon spheroid cell formation.

Stem cells are isolated according to the expression of specific markers, and many stem cell-related genes have been 
reported (8-13). Here, we determined the stemness phenotype using colon stem cell-related genes, such as Lgr5, Klf4, Bmi-1, and Oct-4. In the intestine, Lgr5 is uniquely expressed in stem cells and is switched off in their immediate daughter cells, also known as transit-amplifying cells (8). More recently, Lgr5 expression was observed in human colon cancer, specifically in the regions where CSCs are located, and this gene has therefore been added to the list of colon cancer stem cell markers (9). Klf4 is highly expressed in colon CSC-enriched cells and is essential for maintaining CSC characteristics (10). Bmi-1 is crucial for the self-renewal of stem cells, and ablation of Bmi-1-positive cells leads to crypt loss, suggesting that Bmi-1 is an intestinal stem cell marker (11). In addition, Oct-4 overexpressing colon cancer cells exhibit CSC characteristics, and Oct-4 has been suggested to play an important role in colon CSC survival (12). Moreover, the introduction of two essential transcription factors (Oct-4 and Klf4) that regulate stemness/ differentiation and self-renewal has the potential to reprogram differentiated epithelial cells into induced pluripotent stem cells. This potential reveals these genes as important targets for anticancer drug development (13).

Recently, IL- 6 has been identified as a multifunctional cytokine that participates in disease responses during inflammation, myocardial infarction, autoimmune disorders, and cancer. IL-6 interacts with a membrane-bound receptor (IL-6 receptor; IL-6R) on target cells and mediates signaling that interferes with many cellular functions, such as cell growth and survival, differentiation, cell mobility and angiogenesis $(14,15)$. IL-6 is produced in many types of carcinoma (16-18) and IL- 6 serum levels can be used as a prognostic indicator for colorectal cancer $(19,20)$.

The signal transducer and activator of transcription (STAT) family of transcription factors is activated by many cytokines (including IL-6) and growth factors (EGF and FGF) and promotes transcription in the nucleus. STAT3 has several important roles in tumorigenesis, and the constitutive activation of STAT3 has been shown to confer resistance to chemotherapy-induced apoptosis (21). In particular, the activation of STAT3 in colon CSC-like cells plays a role in the maintenance of cell survival and tumor-forming capacity (22). Studies have also indicated that inhibition of STAT3 activity may serve as an attractive therapeutic approach for colorectal cancer. However, IL-6 signaling and especially STAT3 activation are also involved in stem cell differentiation $(23,24)$. Additionally, IL-6 regulates the Notch 3-dependent signaling pathway, and Notch 3 signaling has been shown to be important in maintaining mammosphere survival (25) and the stem cell phenotype in medulloblastoma (26). Because CSC targeting has been proposed as an effective cancer therapy, the pivotal signals responsible for CSC biology must be clarified. However, the effects of IL- 6 and the mediation of its downstream Notch and STAT3 signaling in colon cancer stemness remain unclear.

This study demonstrated that IL-6 played a pivotal role in colon cancer stem-like properties, and moreover, STAT3 inhibition enhanced stem cell-related gene expression, including Notch 3, and chemoresistance, suggesting the enhancement of stem-like cell properties. These results indicated that an anti-IL-6 antibody or Notch 3 inhibition better targets CSCs compared with STAT3 inhibition.

\section{Materials and methods}

Cells and culture conditions. WiDr cells which are p53 mutant human colorectal cell lines, were obtained from the American Type Culture Collection (Rockville, MD, USA). Cells were grown in RPMI-1640 medium (Sigma-Aldrich, St. Louis, MO, USA) supplemented with $10 \%$ FBS and 1\% antibiotic/ antimycotic in tissue culture dishes in a humidified incubator at $37^{\circ} \mathrm{C}$ in an atmosphere of $95 \%$ air and $5 \%$ carbon dioxide. The culture medium was changed twice weekly, and the cells were passaged using 5\% trypsin/EDTA under attached conditions.

Treatment with IL-6, an anti-human IL-6R antibody, and $5-F U$. To investigate the effects of exogenous IL-6 treatment, the spheroid-forming cells were treated with $50 \mathrm{ng} / \mathrm{ml}$ of IL-6 (Acris, San Diego, CA, USA) for $24 \mathrm{~h}$. To investigate the role of endogenous and exogenous IL-6, the spheroid-forming cells were treated with $100 \mu \mathrm{g} / \mathrm{ml}$ of the anti-human IL-6R antibody MRA (tocilizumab), provided by Chugai Pharmaceutical Co., Ltd. (Tokyo, Japan), for $24 \mathrm{~h}$ in the presence or absence of IL-6. To measure 5-FU sensitivity, the spheroid-forming cells were treated for 3 days with 5-FU (40 $\mu \mathrm{g} / \mathrm{ml})$ (Sigma-Aldrich) and IL-6 and/or MRA.

Colon spheroid formation. Colon spheroids were generated by incubating a limited number of parental WiDr cells at a concentration of $1 \times 10^{4}-1 \times 10^{5} / \mathrm{ml}$ in serum-free stem cell medium (SCM) containing DMEM/Ham's F12 (Sigma-Aldrich) supplemented with B27 (Life Technologies, Gaithersburg, MD, USA), $20 \mathrm{ng} / \mathrm{ml}$ EGF and $10 \mathrm{ng} / \mathrm{ml}$ FGF (Sigma-Aldrich) in untreated dishes or 96-well untreated plate. Experimental procedures were performed after 5 days of spheroid-forming culture. Cell proliferation was assessed using the 3-(4, 5-dimethylthiazol2yl)-2, 5-diphenyltetrazolium bromide (MTT) assay (Promega Corp., Madison, WI, USA). Dye solution (15 $\mu \mathrm{l}$ ) was added to each well for 4 -h incubation at $37^{\circ} \mathrm{C}$, and the solubilization solution was added to the wells to solubilize the formazan product, then after 1-h incubation, mixed using multichannel pipette. The absorbance was measured at $570 \mathrm{~nm}$ using a microplate spectrophotometer.

RT-PCR analysis. The mRNA levels of IL-6, IL-6R, Oct-4, Bmi-1, Klf4, and Notch 3 were quantified by reverse transcriptase polymerase chain reaction (RT-PCR). Total RNA was extracted using the Isogen-LS reagent (Invitrogen) from control cells cultured in adherent conditions and colon spheroids cultured in suspension conditions. The RNA concentration and purity were determined using absorbance measurements at 260 and $280 \mathrm{~nm}$, and $5 \mu \mathrm{g}$ RNA was reverse transcribed using SuperScript II Reverse Transcriptase (Invitrogen). The primers used in RT-PCR were as follows: IL-6, forward 5'-AGTGAGGAACAAGCCAGAGC-3' and reverse 5'-GCGC AGAATGAGATGAGTTGT-3'; IL-6R, forward 5'-CCTGCC AACATCACAGTCACT-3' and reverse 5'-TTTGACCGTTCA GCCCGA-3'; Oct-4, forward 5'-GATGGCGTACTGTGGG CCC-3' and reverse 5'-TGGGACTCCTCCGGGTTTTG-3'; Bmi-1, forward 5'-CCAGGGCTTTTCAAAAATGA-3' and reverse 5'-CCGATCCAATCTGTTCTGGT-3'; Klf4, forward 5'-ATGACCGACGGGCTGCCGTAC-3' and reverse 5'-CTA 
GGCAGGGAGTCCGCTCC-3'; Notch 3, forward 5'-TCAGGC TCTCACCCTTGG-3' and reverse 5'-AGTCACTGGCACGGT TGTAG-3'; GAPDH, forward 5'-CGTCTTCACCACCATGG AGA-3' and reverse 5'-CGGCCATCAC GCCACAGTTT-3'; and Hes3, forward 5'-TGGAGAAGGCCGACATCCTG-3' and reverse 5'-CCGCTGCCGACCTCATCTCC-3'. The thermal cycling conditions were: an initial 5 -min incubation at $94^{\circ} \mathrm{C}$ followed by 40 cycles of $94^{\circ} \mathrm{C}$ for $30 \mathrm{sec}, 60^{\circ} \mathrm{C}$ for $1 \mathrm{~min}$, and $72^{\circ} \mathrm{C}$ for $30 \mathrm{sec}$ and a final $10-\mathrm{min}$ incubation at $72^{\circ} \mathrm{C}$. The PCR products were separated by electrophoresis on a $2 \%$ $(0.02 \mathrm{~g} / \mathrm{ml})$ agarose gel.

Antibodies. The following antibodies were used for western blotting: rabbit anti-LGR5/GPR49 (Abgent, San Diego, CA, USAAP2745d), rabbit anti-STAT3 (Cell Signaling Technology, Inc., Danvers, MA, USA), rabbit anti-phospho-STAT3 (Cell Signaling Technology, Inc.), and rabbit anti-ABCG2 (Cell Signaling Technology, Inc.).

Western blotting. For all western blot analyses, proteins were harvested from adherent cells and colon spheroids. The protein concentrations were determined using a bicinchoninic acid protein assay kit (Pierce Biotechnology, Inc., Rockford, IL, USA). Protein samples for western blotting were boiled after the addition of denaturing sample buffer. Proteins were separated using SDS-PAGE on 6 and 10\% gels and transferred to polyvinylidene difluoride (PVDF) membranes (Millipore) by electroblotting. Antibodies were diluted in TBS and Tween-20 (TBST) with 5\% non-fat dry milk for $1 \mathrm{~h}$ at room temperature to block the residual free protein binding sites on the PVDF membranes. Membranes were incubated at $4^{\circ} \mathrm{C}$ overnight with primary antibodies, subsequently washed with TBST, and incubated with an appropriate horseradish peroxidaseconjugated secondary antibody (Amersham Biosciences) for $1 \mathrm{~h}$ at room temperature. After repeating the TBST washing step, immunoblots were developed using enhanced chemiluminescence, and the luminescence was visualized with X-ray film.

siRNA transfection. siRNA directed against Notch 3 (Stealth select 3 RNAi set) mRNA and appropriate control scrambled siRNAs were purchased from Invitrogen (Darmstadt, Germany). siRNA transfection in colon spheroids was performed by mixing $1 \mu \mathrm{g}$ siRNA in vitro with the JET-PEI reagent (Poly plus Transfection) for 2 days.

Cell cycle analysis. Spheroids were collected and dispersed into single-cell suspensions by trypsinization, pelleted by centrifugation at $1,300 \mathrm{rpm}$ for $3 \mathrm{~min}$, and prepared as a single-cell suspension in $1 \mathrm{ml}$ phosphate-buffered saline (PBS). The solution was stained according to the manufacturer's instructions of Cycle TEST TM Plus DNA Reagent kit (BD Biosciences, San Jose, CA, USA) and analyzed for ploidy using a FACSCanto ${ }^{\mathrm{TM}}$ flow cytometer with FACS Diva 6.1.3 software (BD Biosciences).

Statistics. Student's t-test was employed to examine the differences between the groups. $\mathrm{p}<0.05$ was considered statistically significant. Data are presented as the mean \pm standard deviation (SD).

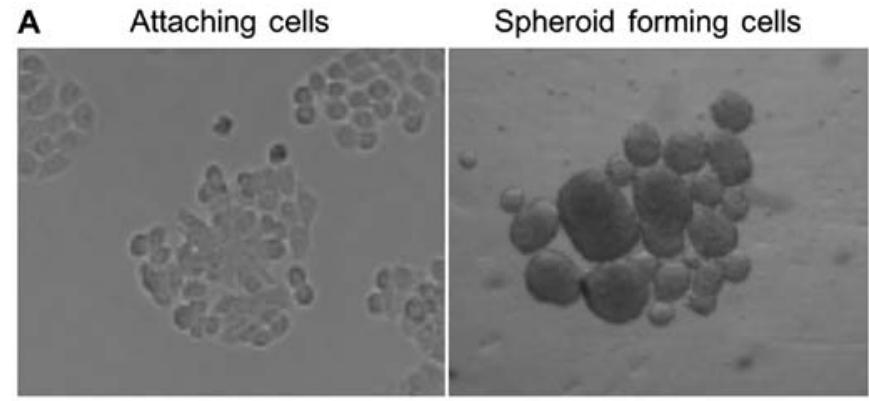

B

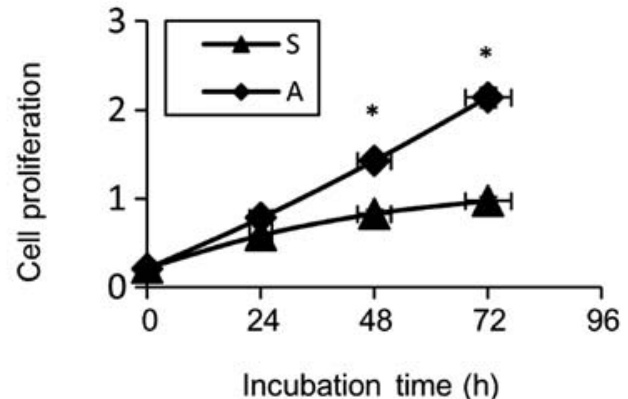

C

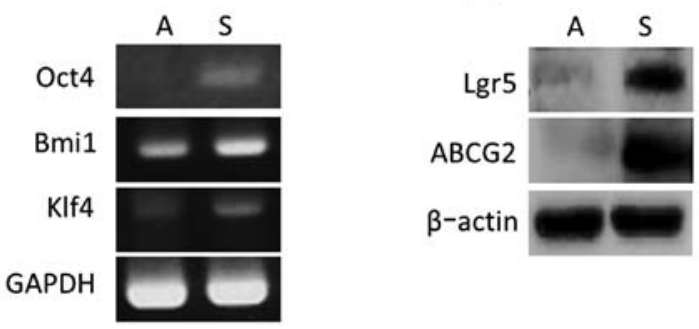

Figure 1. Effects of spheroid formation on colon cancer cell stemness (A) Phase contrast images of WiDr cells cultured under adherent and spheroid-forming conditions. (B) Cell proliferation of adherent (referred to as A) and spheroid-forming cells (referred to as S). (C) Expression of stem cell-related genes in adherent (A) and spheroid-forming cells (S). Cell proliferation was quantified with the MTT assay. ${ }^{*} \mathrm{p}<0.01$.

\section{Results}

Development of stem-like properties. With the exception of the isolation of cells positive for CSC markers, colon CSC-like cells have generally been enriched by culturing primary and developed cancer cell lines in low-serum medium at a relatively low density in the presence of the growth factors EGF and FGF. Recent studies have also revealed that colon spheroids formed from cultured colon cancer cells exhibit lower proliferation potential, higher levels of CSC-associated markers, and higher drug resistance and generate more tumors upon xenotransplantation compared with the corresponding parental cells (27). In agreement with previous observations, our current results revealed that WiDr cells formed colon spheroids in serum-free media under suspension conditions (Fig. 1A). The proliferation of cells cultured for 3 days (72 h) was analyzed with the MTT assay, and we found that colon spheroid-forming cell proliferation was reduced by $\sim 25 \%$ (24 h), 40\% (48 h) and 55\% (72 h) compared with the cells cultured under adherent conditions (Fig. 1B). Moreover, higher expression levels of stem cell-related genes, such as Lgr5, Oct-4, Bmi-1, and Klf4, were observed in spheroid-forming cells (Fig. 1C). Higher expression levels of ABCG2, a member of the ATP binding cassette (ABC) transporter superfamily, 
A
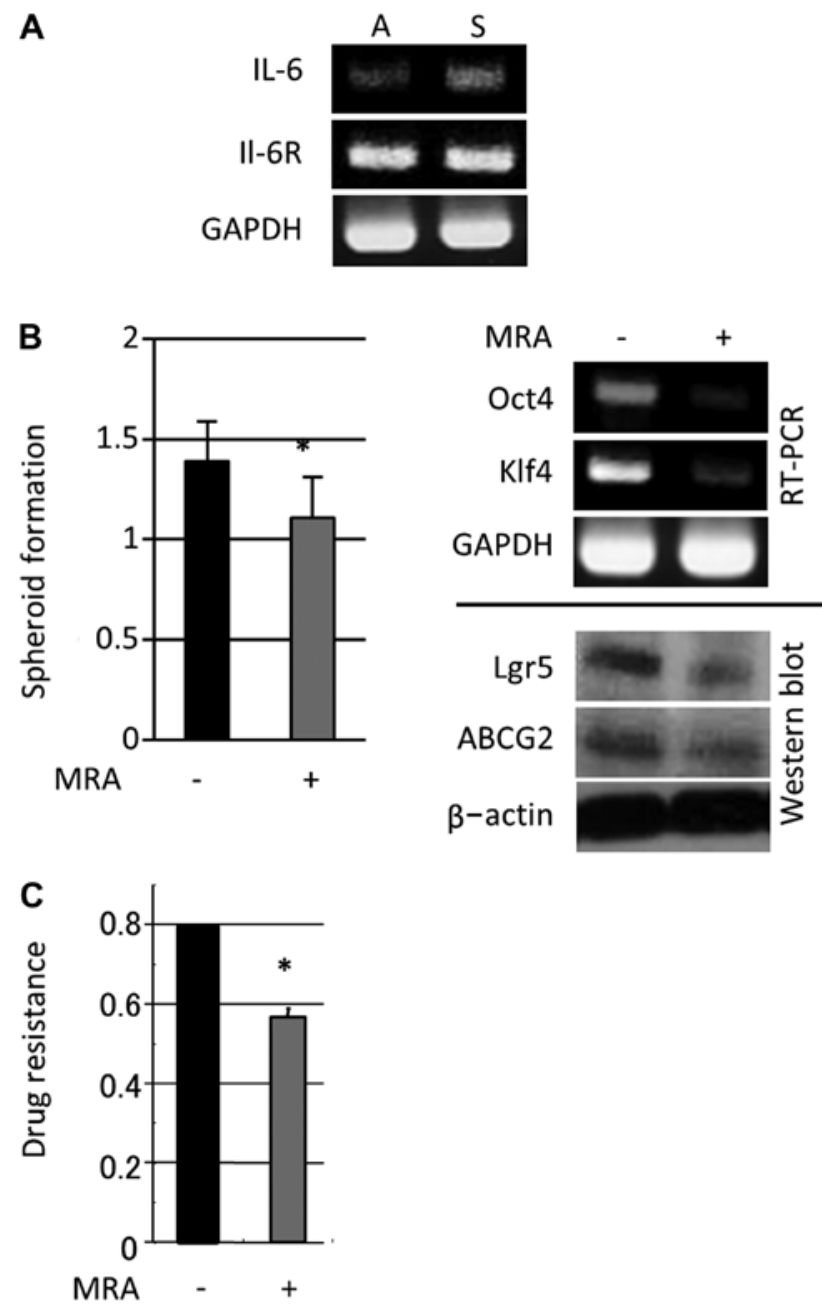

Figure 2. The role of endogenous IL-6 expression in colon cancer stemness. (A) IL-6 and IL-6R expression in adherent (A) and spheroid-forming cells (S). (B) Spheroid-forming ability and the expression of stem cell-related genes in the presence or absence of MRA. (C) Drug resistance of spheroid-forming cells treated with 5-FU in the presence or absence of MRA. Drug resistance was quantified by measuring cell survival with the MTT assay. " $\mathrm{p}<0.01$.

was also observed in spheroid-forming cells compared with adherent cells (Fig. 1C). This ABCG2 upregulation has also been observed in less differentiated tumors and CSCs and is predictive of poor prognosis and impaired responses to chemotherapy (28).

Biological effects of endogenous IL-6 expression on the stemlike properties of colon cancer cells. To assess the involvement of IL-6 in colon cancer cell spheroid formation, we investigated IL-6 and IL-6R expression in the context of WiDr (low IL-6 expression but high IL-6R expression)-derived colon cancer spheroids. Higher levels of IL-6 mRNA were observed in spheroid-forming cells compared with adherent cells; however, cells cultured in both adherent and spheroid-forming conditions expressed similar IL-6R levels (Fig. 2A). Moreover, $24 \mathrm{~h}$ of MRA administration $(100 \mu \mathrm{g} / \mathrm{ml})$ led to a substantial reduction in spheroid formation and reduced the expression of stem cell-related genes (Lgr5, Oct-4 and Klf4) and ABCG2 (Fig. 2B). Additionally, MRA significantly reduced spheroidforming cell proliferation after treatment with $5-\mathrm{FU}(40 \mu \mathrm{g} / \mathrm{ml})$ (Fig. 2C). These data indicated that IL-6 expression induced
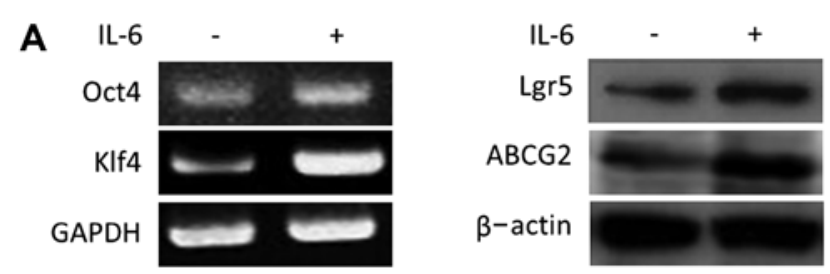

B
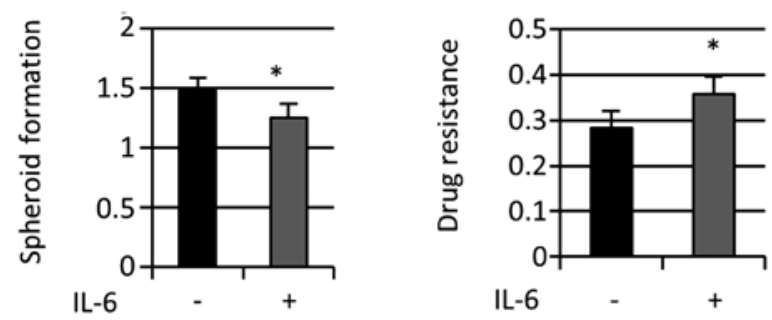

C

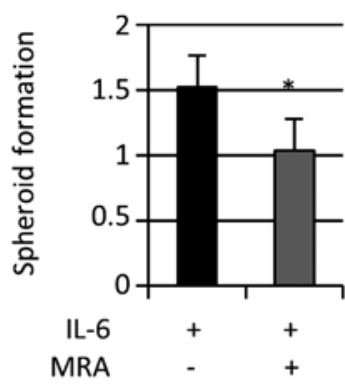

D
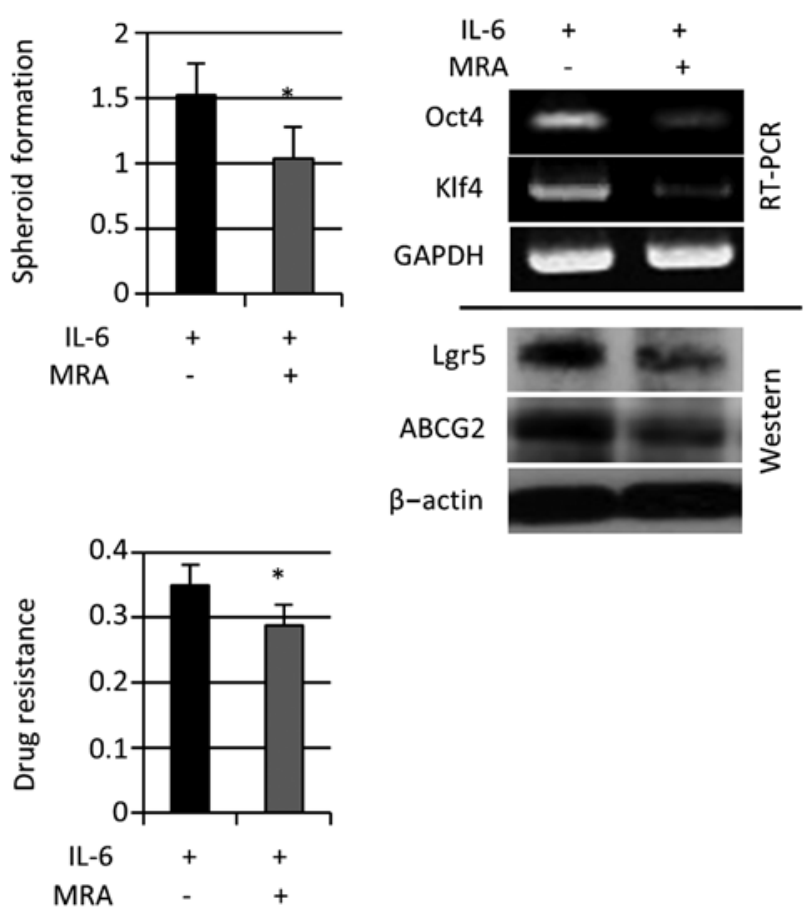

Figure 3. Effects of exogenous IL-6 administration on stemness. (A) Stem cell-related gene expression in the presence or absence of exogenous IL-6. (B) Spheroid-forming ability and drug resistance of spheroid-forming cells treated with 5-FU in the presence or absence of exogenous IL-6. (C) Spheroid-forming ability and the stem cell-related gene expression in spheroid-forming cells in the presence of exogenous IL-6 with or without MRA. (D) Drug resistance of spheroid-forming cells treated with 5-FU in the presence of exogenous IL-6 with or without MRA. Drug resistance was evaluated by determining cell survival with the MTT assay. ${ }^{*} \mathrm{p}<0.01$.

during spheroid-forming culture conditions had a significant impact on colon CSC-like properties, spheroid formation, stem cell-related gene expression and 5-FU resistance.

Effects of exogenous IL-6 treatment on stemness. In colorectal cancer tissue, IL-6 is secreted not only by tumor cells but also by stromal cells, including fibroblasts and immune cells (29). To investigate the effects of exogenous IL-6 on colon cancer stemness, we treated spheroid-forming cells with exogenous IL-6 $(50 \mathrm{ng} / \mathrm{ml})$ for $24 \mathrm{~h}$. Stem cell-related gene expression (Lgr5, Oct-4, and Klf4) and ABCG2 expression increased (Fig. 3A), and 5-FU resistance was enhanced; however, 
A

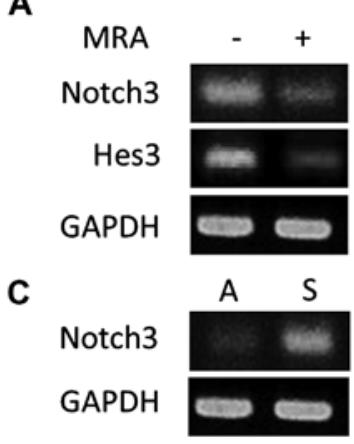

D

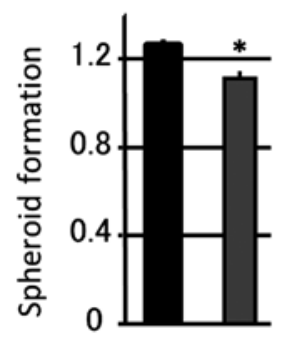

Notch3 siRNA - +

E

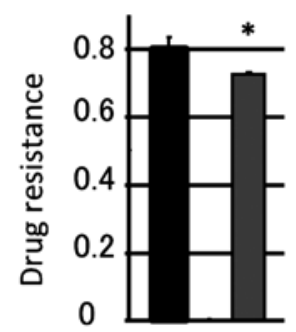

Notch3 siRNA

Figure 4. The role of Notch 3 expression in stemness and IL-6 signaling. (A) Notch 3 and Hes 3 expression in spheroid-forming cells in the presence or absence of MRA. (B) Notch 3 and Hes 3 expression in spheroid-forming cells in the presence of exogenous IL-6 with or without MRA. (C) Notch 3 expression in adherent (A) and spheroid-forming cells (S). (D) Spheroidforming ability and stem cell-related gene expression in spheroid-forming cells treated with the negative control siRNA and Notch 3 siRNA. (E) Drug resistance of spheroid-forming cells treated with 5-FU and negative control siRNA or Notch 3 siRNA. Drug resistance was evaluated by determining cell survival with the MTT assay. " $\mathrm{p}<0.01$.

spheroid-forming cell proliferation estimated with the MTT assay decreased (Fig. 3B). In addition, stem cell-related gene expression and spheroid formation decreased after the addition of MRA (100 $\mu \mathrm{g} / \mathrm{ml})$ for $24 \mathrm{~h}$ (Fig. 3C). MRA treatment also reduced 5-FU resistance observed during colon spheroid formation that was enhanced by exogenous IL-6 (Fig. 3D). Taken together, these results suggested that exogenous IL-6 further enhanced the induction of colon cancer stem-like properties.

The role of Notch 3 in IL-6-induced stemness. The Notch pathway is a critical downstream target of IL-6, and IL-6 treatment triggers the upregulation of Notch 3 expression and promotes primary human mammosphere formation (20). In this study, we found that $24 \mathrm{~h}$ of MRA administration $(100 \mu \mathrm{g} / \mathrm{ml})$ downregulated Notch 3 mRNA expression and the expression of its downstream target gene Hes3 in colon cancer spheroidforming cultures (Fig. 4A). Moreover, the administration of
A

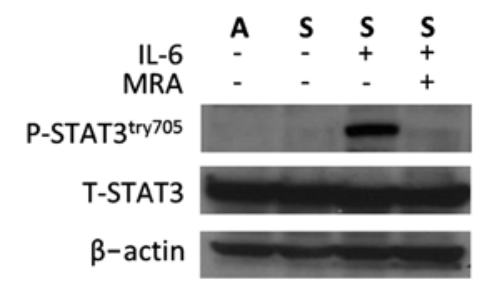

B

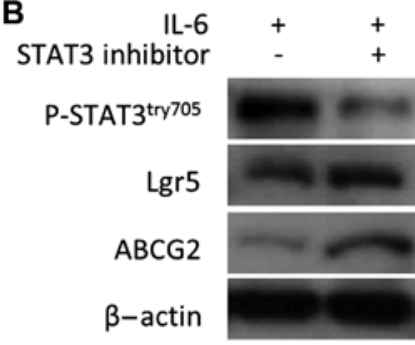

C

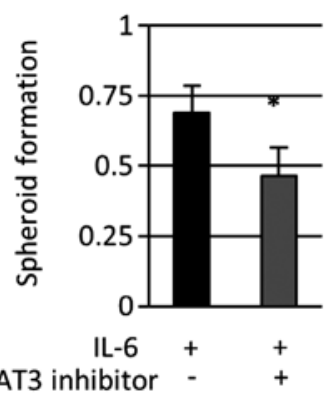

D
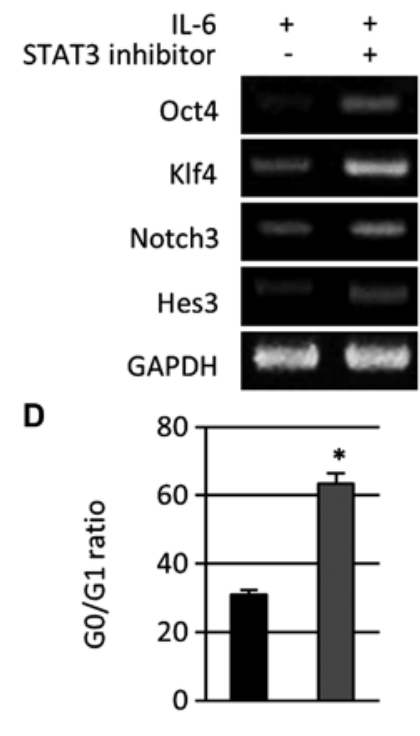

IL-6

STAT3 inhibitor - $\quad+$

Figure 5. Effects of STAT3 activity on stemness. (A) STAT3 activity in adherent cells (A), spheroid-forming cells (S), spheroid-forming cells treated with exogenous IL-6, and spheroid-forming cells treated with exogenous IL-6 combined plus MRA. (B) Stem cell-related gene expression in spheroidforming cells in the presence of exogenous IL-6 with or without a STAT3 inhibitor. (C) Spheroid formation in the presence of exogenous IL-6 with or without a STAT3 inhibitor. (D) Ratio of spheroid-forming cells in the $G_{0} / G_{1}$ phase in the presence of exogenous IL- 6 with or without a STAT3 inhibitor. Spheroid-forming cells were stained with PI and cell cycle populations were calculated by FACS. ${ }^{\mathrm{p}}<0.01$.

exogenous IL-6 $(50 \mathrm{ng} / \mathrm{ml})$ to colon cancer spheroids for $24 \mathrm{~h}$ upregulated Notch 3 and Hes 3 levels, and MRA treatment also downregulated Notch 3 mRNA expression and Hes 3 expression induced by exogenous IL-6 (Fig. 4B).

In recent years, the Notch signaling pathway has been shown to play a critical role in regulating the balance between cell proliferation, differentiation, and apoptosis in various tissues. In this study, Notch 3 was more highly expressed in colon spheroid-forming cells compared with adherent cells (Fig. 4C), and a blockade of Notch 3 signaling via siRNA induced a marked reduction in colon spheroid self-renewal, the expression of stem cell-related genes (Fig. 4D) and drug resistance (Fig. 4E). These data indicated that Notch 3 signaling is pivotal for upregulating the (endogenous and exogenous) IL-6-dependent induction of stem-like properties.

STAT3 activation in IL-6-induced stemness. The STAT protein family includes various transcription factors that play a role in relaying extracellular signals initiated by cytokines and growth factors from the cytoplasm to the nucleus. As a downstream target of IL-6, higher levels of the active phosphorylated form 
of STAT3 were observed in colon stem-like cells and could be reduced by targeting IL-6 (30). The present study demonstrated that treatment with exogenous IL-6 induced STAT3 phosphorylation at tyrosine residue 705 (Y705), whereas MRA treatment suppressed this phosphorylation event (Fig. 5A). We also treated spheroid-forming cells with $100 \mu \mathrm{M}$ NSC74859, a STAT3 inhibitor, to examine the effect of a STAT3 signaling blockade in colon cancer stemness enhanced by IL- 6 treatment. Two hours of NSC74859 treatment suppressed STAT3 activity and increased the expression of stem cell-related genes (Lgr5, Oct-4 and Klf4), ABCG2, Notch 3 and Hes3 (Fig. 5B); however, this treatment decreased spheroid formation (Fig. 5C). Moreover, STAT3 blockade increased the proportion of spheroid-forming cells in the $\mathrm{G}_{0} / \mathrm{G}_{1}$ phase (Fig. 5D). These results suggested that STAT3 activation enhanced cell proliferation but reduced stemness and that STAT3 inhibition enhanced the development of stemness-specific phenotypes in colon cancer cells.

\section{Discussion}

The existence of CSCs was proposed over 40 years ago (31), and cancer is currently regarded as an aberrant organogenesis supported by a minority of cancer cells termed tumor-initiating cells or CSCs. CSCs exhibit three properties: the capacity for self-renewal, the potential for multilineage differentiation, and cytoprotective characteristics, including low proliferative potential, DNA repair, and high expression of anti-apoptotic factors. Colon cancers also arise from a small population of CSCs through oncogenic transformations (32). Indeed, CSCs are not only the source of the tumor itself but also form the basis for resistance to therapy, which leads to tumor progression, metastasis and tumor recurrence. Recently, clarifying the mechanisms responsible for the maintenance of CSC properties has led to the development of CSC-targeted therapies.

To identify and analyze the function of CSCs, several methods have been developed. The spheroid-formation technique has been used to isolate putative CSC-like cells from brain (33), breast (34), and colon tumors (35), and this type of suspension culture is thought to maintain CSC-like cells in an undifferentiated state, which enables their enrichment. Observing the formation of colon cancer spheroids suggests the presence of functional CSCs in colon cancer-derived cell lines (36). In this study, we used a suspension culture approach to induce spheroid formation in the colon cancer cell line WiDr and observed that spheroid formation reduced cell cycle progression, enhanced the expression of stem cellrelated genes (Lgr5, Bmi-1, Klf4 and Oct-4), and increased the expression levels of $\mathrm{ABCG} 2$, which represent traits that have been previously described in CSCs $(37,38)$. However, spheroid growth per se does not ensure the enrichment of only less differentiated cell types (39). During spheroid culture, the progressive upregulation of differentiation genes can be detected (40), and spheroids are composed of cells that express various levels of stem cell markers (41). In this study, such a discrepancy between the proliferation of spheroid-forming cells and the other stemness traits was observed; namely, exogenous IL-6 administration or STAT3 inhibition decreased spheroid-forming cell proliferation but enhanced stem cellrelated gene expression, the $\mathrm{G}_{0} / \mathrm{G}_{1}$ population, and 5-FU resistance, indicating that these treatments induced the formation of spheroids largely composed of stemness-rich cells. It was difficult to show the heterogeneous distribution of stem cell-related gene expression within a single sphere; therefore, these results further highlighted the discrepancy between spheroid growth and stemness.

Several experimental studies have demonstrated that IL-6 promotes tumorigenesis, angiogenesis, metastasis and treatment resistance (42-45). The serum IL-6 levels have also been correlated with disease status and prognosis in patients with various malignant diseases (46-48), and early evidence suggests that IL-6 may play an important role in CSC phenotype and function (49). In pancreatic and lung cancers, stem cells produce a significant amount of IL-6, thereby regulating CSCs characteristics in autocrine and paracrine loops $(44,50)$. In breast cancer, IL-6 secreted from non-stem cells regulates CSC-associated Oct- 4 gene expression and plays a critical role in the conversion of non-stem cells into stem-like cells (46). This study also demonstrated that IL-6, whose expression was induced in colon cancer spheroid-forming cells, was critical to the induction and maintenance of colon cancer stem-like properties, thus indicating the existence of positive feedback.

IL-6 has been implicated as an important activator of Jagged-1/Notch signaling in spheroid-forming cells (51). In this study, we observed that Notch 3 expression was markedly enhanced in colon cancer spheroid-forming cells compared with adherent cells. Furthermore, Notch 3 expression was upregulated by IL- 6 administration, and an anti-human IL-6R monoclonal antibody suppressed Notch 3 expression. Moreover, the inhibition of Notch 3 expression by siRNA suppressed spheroid formation, the expression of stem cellrelated genes and resistance to 5-FU treatment. Previous studies have demonstrated that Notch and its downstream molecules function as oncogenes in several cancers, including bone, brain, breast, lung, pancreatic and colon cancers $(52,53)$. The Notch pathway has also been reported to be highly active in CSCs and essential for the self-renewal capacity and tumorigenicity of these cells $(26,47,48)$. Several reports have further suggested that Notch 1 is involved in the stem cell biology of colorectal cancer $(54,55)$, and Notch 3 has also been reported to play an important role in stemness in other organs $(25,56,57)$. In this study, however, no significant changes in Notch 1 expression were detected after spheroid formation or IL-6 administration.

The JAK-STAT pathway is the major IL-6 downstream signaling pathway, and IL-6 is involved in the activation of oncogenic STAT3 in adenocarcinomas (51). Moreover, constitutive activation of STAT3 is frequently detected in primary human cancer cells, including colorectal cancer cells (58). Activated phosphorylated STAT targets the expression of numerous critical genes (c-myc, survivin, cyclin D1, Bcl-2, Bcl-xl, HIF-1 $\alpha$, and VEGF) and regulates cell cycle progression, proliferation, invasion, and survival $(59,60)$. Persistent STAT3 activation is also associated with enhanced proliferation, the invasion of colorectal cancer cells in vitro, and tumor growth in colorectal tumor in vivo models, and STAT3 inhibition induces apoptosis and reduces tumor cell invasion (61). These reports indicate that constitutive STAT3 activation is one of the important pathways contributing to oncogenesis in colorectal cancer, and this signaling pathway is thus an attrac- 
tive therapeutic target. In addition, stem cell marker-positive cells express higher levels of the active phosphorylated form of STAT3 compared with stem cell marker-negative cells (30), and in colon cancer stem-like cells, blocking STAT3 signaling has been shown to suppress cancer stem-like cell growth and downregulate the expression of many genes related to cancer cell proliferation, survival, and angiogenesis. These reports indicate that STAT3 activation plays an important role in CSC biology.

Previous studies showed that STAT3 activation requires phosphorylation of STAT3 protein both on Tyr705 and Ser727 residues in response to stimulation by cytokines and growth factors (62-64). CD $34^{+}$cells purified from chronic myelogenous leukemia patients also had increased phosphorylation levels on Tyr705 and Ser727 (65). However, the exact mechanism of these differential regulations of STAT3 Tyr705 and Ser727 is not known. Other studies have suggested that phosphorylation of STAT Ser727 is independent of STAT3 Tyr705 phosphorylation (66). STAT3 Ser727 phosphorylation was also stimulated by insulin, anisomycin, tumor necrosis factor- $\alpha$, or arsenite and, to a weaker extent, by $\mathrm{NaCl}$, okadaic acid, or lipopolysaccharide; however, Tyr705 phosphorylation was not detected (67-69). Regarding stemness and differentiation, Tyr705 and Ser727 have opposing roles. STAT3 Ser727 phosphorylation has been reported to be important for the maintenance of the stem cell properties of neural stem/progenitor cells $(70,71)$. In contract, STAT3 Tyr705 phosphorylation has also been shown to induce the differentiation of stem cells derived from other organs (72-74). In this study, endogenous IL-6 was induced by spheroid formation and was critical in spheroid formation through an autocrine loop, but STAT3 phosphorylation at Ser727 or Tyr705 was not observed in spheroid-forming cells, indicating that in WiDr cells, the IL-6/STAT3 pathway was not involved in the induction/maintenance of stemness. On the other hand, exogenous IL-6 treatment activated STAT3 (Tyr705) phosphorylation, decreased the proliferation of spheroid-forming cells, and enhanced the expression of stemrelated genes and 5-FU resistance. These results indicated that exogenous IL-6 induced stemness via Notch 3 expression rather than differentiation by Tyr705 phosphorylation. A STAT3 specific inhibitor has been reported to suppress Tyr705 phosphorylation and induce stemness (75), and in this study, blocking STAT3 activity in WiDr cells treated with exogenous IL-6 suppressed Tyr705 phosphorylation and increased the proportion of resting $\mathrm{G}_{0} / \mathrm{G}_{1}$ cells and other CSC characteristics, such as the expression of stem cell-related genes and 5-FU resistance, indicating the induction of CSC traits.

Various reports have documented the relationship between STAT3 activation and Notch signaling, another downstream target of IL-6. For example, STAT3 has been reported to be involved in spheroid formation and the expression of stem markers via Notch expression (76), and inversely, Notch is reported to induce stem marker expression via STAT3 activation (77). STAT3 has also been found to play dual tumor suppressive and oncogenic roles in glial malignancy depending on the mutational profile of tumors. In this study, we observed that Notch 3 expression was unexpectedly upregulated by STAT3 inhibition, indicating the existence of negative crosstalk between IL-6 downstream signals and the potential for stemness mediated by STAT3 inhibition and
Notch 3 upregulation. These findings demonstrate that in this situation, STAT3 de-phosphorylation (Tyr705) mediates the survival effects of Notch activation.

In this study using WiDr cells, IL-6 played a pivotal role in stemness of colon cancer cells, as previously reported in other organs $(46,49,50)$. However, inhibition of STAT3, a well-known suppression of the IL-6 pathway enhanced rather than inhibited stemness, and an anti-IL-6 receptor monoclonal antibody or Notch 3 inhibition effectively suppressed stemness. These results demonstrate the rationale for tailor-made therapy (78). We have developed an effective method, cancer tissue-originated spheroid (CTOS), to purify cancer cells from colon cancer tissues and culture and expand these cells in in vitro and in vivo systems while retaining the features of parental tumors (79). It is desirable to analyze the precise characteristics of cancer cells in each individual case, by employing reproducible methods such as these, and to determine the most suitable remedy.

In conclusion, our data demonstrate that IL- 6 plays a pivotal role in the biology of colon CSCs via Notch 3 signaling rather than STAT3 signaling. Furthermore, an anti-human IL-6 receptor monoclonal antibody downregulated this signaling pathway, suppressed the expression of colon stem markers and significantly increased chemosensitivity. These data therefore indicate that an anti-human IL-6 receptor monoclonal antibody or Notch signaling inhibition may represent an effective antitumor treatment approach when used in combination with conventional chemotherapies.

\section{Acknowledgements}

We thank Chugai Pharmaceutical Co., Ltd. (Tokyo, Japan) for kindly supplying MRA (tocilizumab), a humanized antihuman IL-6 receptor monoclonal antibody.

\section{References}

1. Gabrilovich D: Mechanisms and functional significance of tumour-induced dendritic-cell defects. Nat Rev Immunol 4: 941-952, 2004

2. Fang DD, Kim YJ, Lee CN, et al: Expansion of CD133 (+) colon cancer cultures retaining stem cell properties to enable cancer stem cell target discovery. Br J Cancer 102: 1265-1275, 2010.

3. Dick JE: Stem cell concepts renew cancer research. Blood 112: 4793-4807, 2008.

4. Sukach AN and Ivanov EN: Formation of spherical colonies as a property of stem cells. Tsitologiia 49: 916-922, 2007 (In Russian).

5. Singh SK, Clarke ID, Terasaki M, Bonn VE, Hawkins C, Squire J and Dirks PB: Identification of a cancer stem cell in human brain tumors. Cancer Res 63: 5821-5828, 2003.

6. Farnie G, Clarke RB, Spence K, Pinnock N, Brennan K, Anderson NG and Bundred NJ: Novel cell culture technique for primary ductal carcinoma in situ: role of Notch and epidermal growth factor receptor signaling pathways. J Natl Cancer Inst 99: 616-627, 2007.

7. Vermeulen L, Todaro M, de Sousa Mello F, Sprick MR, Kemper K, Perez Alea M, Richel DJ, Stassi G and Medema JP: Single-cell cloning of colon cancer stem cells reveals a multilineage differentiation capacity. Proc Natl Acad Sci USA 105: 13427-13432, 2008.

8. van der Flier LG, van Gijn ME, Hatzis P, et al: Transcription factor achaetescute-like 2 controls intestinal stem cell fate. Cell 136: 903-912, 2009.

9. Barker N, van Es JH, Kuipers J, et al: Identification of stem cells in small intestine and colon by marker gene Lgr5. Nature 449: 1003-1007, 2007

10. Leng Z, Tao K, Xia Q, Tan J, Yue Z, Chen J, Xi H, Li J and Zheng H: Kruppel-like factor 4 acts as an oncogene in colon cancer stem cell-enriched spheroid cells. PLoS One 8: e56082, 2013. 
11. Sangiorgi $\mathrm{E}$ and Capecchi MR: Bmi1 is expressed in vivo in intestinal stem cells. Nat Genet 40: 915-920, 2008.

12. Wen K, Fu Z, Wu X, Feng J,Chen W and Qian J: Oct-4 is required for an antiapoptotic behavior of chemoresistant colorectal cancer cells enriched for cancer stem cells: effects associated with STAT3/Survivin. Cancer Lett 333: 56-65, 2013.

13. Takahashi K, Tanabe K, Ohnuki M, Narita M, Ichisaka T, Tomoda K and Yamanaka S: Induction of pluripotent stem cells from adult human fibroblasts by defined factors. Cell 131: 861-872, 2007.

14. Ishihara $\mathrm{K}$ and Hirano T: IL- 6 in autoimmune disease and chronic inflammatory proliferative disease. Cytokine Growth Factor Rev 13: 357-368, 2002.

15. Nian M, Lee P, Khaper N and Liu P: Inflammatory cytokines and postmyocardial infarction remodeling. Circ Res 94: 1543-1553, 2004.

16. Wu CW, Wang SR, Chao MF, Wu TC, Lui WY, P'eng FK and Chi CW: Serum interleukin-6 levels reflect disease status of gastric cancer. Am J Gastroenterol 91: 1417-1422, 1996.

17. Koontongkaew S, Amornphimoltham $\mathrm{P}$ and Yapong B: Tumorstroma interactions influence cytokine expression and matrix metalloproteinase activities in paired primary and metastatic head and neck cancer cells. Cell Biol Int 33: 165-173, 2009.

18. Burger RA, Grosen EA, Ioli GR, et al: Spontaneous release of interleukin- 6 by primary cultures of lymphoid and tumor cell populations purified from human ovarian carcinoma. J Interferon Cytokine Res 15: 255-260, 1995.

19. Knupfer H and Preiss R: Serum interleukin-6 levels in colorectal cancer patients - a summary of published results. Int J Colorectal Dis 25: $135-140,2010$.

20. Hsu CP and Chung YC: Influence of interleukin- 6 on the invasiveness of human colorectal carcinoma. Anticancer Res 26: 4607-4614, 2006.

21. Corvinus FM, Orth C, Moriggl R, et al: Persistent STAT3 activation in colon cancer is associated with enhanced cell proliferation and tumor growth. Neoplasia 7: 545-555, 2005.

22. Lin L, Fuchs J, Li C, Olson V, Bekaii-Saab T and Lin J: STAT3 signaling pathway is necessary for cell survival and tumor sphere forming capacity in $\mathrm{ALDH}(+) / \mathrm{CD} 133(+)$ stem cell-like human colon cancer cells. Biochem Biophys Res Commun 416: 246-251, 2011.

23. Cheng X, Jin G, Zhang X, Tian M and Zou L: Stage-dependent STAT3 activation is involved in the differentiation of rat hippocampus neural stem cells. Neurosci Lett 493: 18-23, 2011.

24. Luo Y, Mughal MR, Ouyang TG, Jiang H, Luo W, Yu QS, Greig NH and Mattson MP: Plumbagin promotes the generation of astrocytes from rat spinal cord neural progenitors via activation of the transcription factor Stat3. J Neurochem 115: 1337-1349, 2010.

25. Sansone P, Storci G, Tavolari S, et al: IL-6 triggers malignant features in mammospheres from human ductal breast carcinoma and normal mammary gland. J Clin Invest 117: 3988-4002, 2007.

26. Fan X, Matsui W, Khaki L, Stearn D, Chun J, Li YM and Eberhart CG: Notch pathway inhibition depletes stem-like cells and blocks engraftment in embryonal brain tumors. Cancer Res 66: 7445-7452, 2006.

27. Fan X, Ouyang N, Teng $\mathrm{H}$ and Yao $\mathrm{H}$ : Isolation and characterization of spheroid cells from the HT29 colon cancer cell line. Int J Colorectal Dis 26: 1279-1285, 2011.

28. Fletcher JI, Haber M, Henderson MJ and Norris MD: ABC transporters in cancer: more than just drug efflux pumps. Nat Rev Cancer 10: 147-156, 2010.

29. Olszewski WL, Kubicka U, Tarnowski W, Bielecki K, Ziolkowska A and Wesolowska A: Activation of human peritoneal immune cells in early stages of gastric and colon cancer. Surgery 141: 212-221, 2007.

30. Lin L, Liu A, Peng Z, Lin HJ, Li PK, Li PK, Li C and Lin J STAT3 is necessary for proliferation and survival in colon cancer-initiating cells. Cancer Res 71: 7226-7237, 2011.

31. Bruce WR and van der Gaag H: A quantitative assay for the number of murine lymphoma cells capable of proliferation in vivo. Nature 199: 79-80, 1963.

32. Willson JK, Bittner GN, Oberley TD, Meisner LF and Weese JL: Cell culture of human colon adenomas and carcinomas. Cancer Res 47: 2704-2713, 1987.

33. Singh SK, Hawkins C, Clarke ID, Squire JA, Bayani J, Hide T, Henkelman RM, Cusimano MD and Dirks PB: Identification of human brain tumour initiating cells. Nature 432: 396-401, 2004.

34. Dey D, Saxena M, Paranjape AN, Krishnan V, Giraddi R, Kumar MV, Mukherjee G and Rangarajan A: Phenotypic and functional characterization of human mammary stem/progenitor cells in long term culture. PLoS One 4: e5329, 2009.
35. Todaro M, Alea MP, Di Stefano AB, et al: Colon cancer stem cells dictate tumor growth and resist cell death by production of interleukin-4. Cell Stem Cell 1: 389-402, 2007.

36. Lopez J, Poitevin A, Mendoza-Martinez V, Perez-Plasencia C and Garcia-Carranca A: Cancer-initiating cells derived from established cervical cell lines exhibit stem-cell markers and increased radioresistance. BMC Cancer 12: 48, 2012.

37. Christgen M, Ballmaier M, Bruchhardt H, von Wasielewski R, Kreipe $\mathrm{H}$ and Lehmann $\mathrm{U}$ : Identification of a distinct side population of cancer cells in the Cal-51 human breast carcinoma cell line. Mol Cell Biochem 306: 201-212, 2007.

38. Han B and Zhang JT: Multidrug resistance in cancer chemotherapy and xenobiotic protection mediated by the half ATP-binding cassette transporter ABCG2. Curr Med Chem Anticancer Agents 4: 31-42, 2004.

39. Lichtenauer UD, Shapiro I, Osswald A, Meurer S, Kulle A, Reincke M, Riepe F and Beuschlein F: Characterization of NCI-H295R cells as an in vitro model of hyperaldosteronism. Horm Metab Res 45: 124-129, 2013.

40. Gabriel E, Schievenbusch S, Kolossov E, Hengstler JG, Rotshteyn T, Bohlen H, Nierhoff D, Hescheler $J$ and Drobinskaya L: Differentiation and selection of hepatocyte precursors in suspension spheroid culture of transgenic murine embryonic stem cells. PLoS One 7: e44912, 2012.

41. Ohata H, Ishiguro T, Aihara Y, et al: Induction of the stem-like cell regulator CD44 by Rho kinase inhibition contributes to the maintenance of colon cancer-initiating cells. Cancer Res 72: 5101-5110, 2012.

42. Scheller J and Rose-John S: Interleukin-6 and its receptor: from bench to bedside. Med Microbiol Immunol 195: 173-183, 2006.

43. Fisman EZ and Tenenbaum A: The ubiquitous interleukin-6: a time for reappraisal. Cardiovasc Diabetol 9: 62, 2010.

44. Yi H, Cho HJ, Cho SM, Jo K, Park JA, Lee SH, Chang BJ, Kim JS and Shin HC: Effect of 5-FU and MTX on the expression of drug-resistance related cancer stem cell markers in nn-small cell lung cancer cells. Korean J Physiol Pharmacol 16: 11-16, 2012.

45. Conze D, Weiss L, Regen PS, Bhushan A, Weaver D, Johnson P and Rincon M: Autocrine production of interleukin 6 causes multidrug resistance in breast cancer cells. Cancer Res 61: 8851-8858, 2001.

46. Kim SY, Kang JW, Song X, Kim BK, Yoo YD, Kwon YT and Lee YJ: Role of the IL-6-JAK1-STAT3-Oct-4 pathway in the conversion of non-stem cancer cells into cancer stem-like cells. Cell Signal 25: 961-969, 2013.

47. Garzia L, Andolfo I, Cusanelli E, et al: MicroRNA-199b-5p impairs cancer stem cells through negative regulation of HES1 in medulloblastoma. PLoS One 4: e4998, 2009.

48. Wang Z, Li Y, Kong D, et al: Acquisition of epithelial-mesenchymal transition phenotype of gemcitabine-resistant pancreatic cancer cells is linked with activation of the notch signaling pathway. Cancer Res 69: 2400-2407, 2009.

49. Levina V, Marrangoni AM, DeMarco R, Gorelik E and Lokshin AE: Drug-selected human lung cancer stem cells: cytokine network, tumorigenic and metastatic properties. PLoS One 3: e3077, 2008.

50. Bao B, Ali S, Ahmad A, et al: Hypoxia-induced aggressiveness of pancreatic cancer cells is due to increased expression of VEGF, IL-6 and miR-21, which can be attenuated by CDF treatment. PLoS One 7: e50165, 2012.

51. Grivennikov S and Karin M: Autocrine IL-6 signaling: a key event in tumorigenesis? Cancer Cell 13: 7-9, 2008.

52. Radtke $\mathrm{F}$ and Raj $\mathrm{K}$ : The role of Notch in tumorigenesis: oncogene or tumour suppressor? Nat Rev Cancer 3: 756-767, 2003.

53. Leong KG and Karsan A: Recent insights into the role of Notch signaling in tumorigenesis. Blood 107: 2223-2233, 2006.

54. Reedijk M, Odorcic S, Zhang H, Chetty R, Tennert C, Dickson BC, Lockwood G, Gallinger S and Egan SE: Activation of Notch signaling in human colon adenocarcinoma. Int J Oncol 33: 1223-1229, 2008

55. Zhang H, Li W, Nan F, Ren F, Wang H, Xu Y and Zhang F: MicroRNA expression profile of colon cancer stem-like cells in HT29 adenocarcinoma cell line. Biochem and Biophys Res Commun 404: 273-278, 2011

56. Sullivan JP, Spinola M, Dodge M, et al: Aldehyde dehydrogenase activity selects for lung adenocarcinoma stem cells dependent on notch signaling. Cancer Res 70: 9937-9948, 2010.

57. McAuliffe SM, Morgan SL, Wyant GA, et al: Targeting Notch, a key pathway for ovarian cancer stem cells, sensitizes tumors to platinum therapy. Proc Natl Acad Sci USA 109: E2939-E2948, 2012. 
58. Ma XT, Wang S, Ye YJ, Du RY, Cui ZR and Somsouk M: Constitutive activation of Stat 3 signaling pathway in human colorectal carcinoma. World J Gastroenterol 10: 1569-1573, 2004.

59. Ashizawa T, Miyata H, lizuka A, et al: Effect of the STAT3 inhibitor STX-0119 on the proliferation of cancer stem-like cells derived from recurrent glioblastoma. Int J Oncol 43: 219-227, 2013.

60. Song X, Wang M, Zhang L, Zhang J, Wang X, Lui W, Gu X and Lv C: Changes in cell ultrastructure and inhibition of JAK1/ STAT3 signaling pathway in CBRH-7919 cells with astaxanthin. Toxicol Mech Methods 22: 679-686, 2012.

61. Lin Q, Lai R, Chirieac LR, et al: Constitutive activation of JAK3/ STAT3 in colon carcinoma tumors and cell lines: inhibition of JAK3/STAT3 signaling induces apoptosis and cell cycle arrest of colon carcinoma cells. Am J Pathol 167: 969-980, 2005.

62. Decker T and Kovarik P: Serine phosphorylation of STATs. Oncogene 19: 2628-2637, 2000.

63. Wen Z, Zhong Z and Darnell JE Jr: Maximal activation of transcription by Stat 1 and Stat 3 requires both tyrosine and serine phosphorylation. Cell 82: 241-250, 1995.

64. Takaoka A, Tanaka N, Mitani Y, et al: protein tyrosine kinase Pyk2 mediates the Jak-dependent activation of MAPK and Stat1 in IFN-gamma, bu not IFN-alpha, signaling. EMBO J 18 : 2480-2488, 1999.

65. Coppo P, Dusanter-Fourt I, Millot G, et al: Constitutive and specific activation of STAT3 by BCR-ABL in embryonic stem cells. Oncogene 22: 4102-4110, 2003.

66. Zhang Y, Liu G and Dong Z: MSK1 and JNKs mediate phosphorylation of STAT3 in UVA-irradiated mouse epidermal JB6 cells. J Biol Chem 276: 42534-42542, 2001.

67. Lim CP and Cao X: Serine phosphorylation and negative regulation of Stat 3 by JNK. J Biol Chem 274: 31055-31061, 1999.

68. Ceresa BP and Pessin JE: Insulin stimulates the serine phosphorylation of the signal transducer and activator of transcription (STAT3) isoform. J Biol Chem 271: 12121-12124, 1996.

69. Ceresa BP, Horvath CM and Pessin JE: Signal transducer and activator of transcription-3 serine phosphorylation by insulin is mediated by a Ras/Raf/MEK-dependent pathway. Endocrinology 138: 4131-4137, 1997.
70. Androutsellis-Theotokis A, Leker RR, Soldner F, et al: Notch signaling regulates stem cell numbers in vitro and in vivo. Nature 442: 823-826, 2006.

71. Nagao M, Sugimori M and Nakafuku M: Cross talk between notch and growth factor/cytokine signaling pathways in neural stem cells. Mol Cell Biol 27: 3982-3994, 2007.

72. Rajan P and Mckay RD: Multiple routes to astrocytic differentiation in the CNS. J. Neurosci 18: 3620-3629, 1998

73. Kwak YD, Dantuma E, Merchant S, Bushnev S and Sugaya K: Amyloid-beta precursor protein induces glial differentiation of neural progenitor cells by activation of the IL-6/gp130 signaling pathway. Neurotox Res 18: 328-338, 2010.

74. Peng H, Sun L, Jia B, Lan X, Zhu B, Wu Y and Zheng J: HIV-1-infected and immune-activated macrophages induce astrocytic differentiation of human cortical neural progenitor cells via the STAT3 pathway. PLoS One 6: e19439, 2011.

75. Luo M, Liu Z, Chen G, Hao H, Lu T, Cui Y, Lei M, Verfaillie CM and Liu Z: High glucose enhances TGF-betal expression in rat bone marrow stem cells via ERK1/2-mediated inhibition of STAT3 signaling. Life Sci 90: 509-518, 2012.

76. Yoshimatsu T, Kawaguchi D, Oishi K, Takeda K, Akira S, Masuyama N and Gotoh Y: Non-cell-autonomous action of STAT3 in maintenance of neural precursor cells in the mouse neocortex. Development 133: 2553-2563, 2006.

77. Choi B, Chun E, Kim SY, Kim M, Lee KY and Kim SJ: Notch-induced hIL-6 production facilitates the maintenance of self-renewal of hCD34 ${ }^{+}$cord blood cells through the activation of Jak-PI3K-STAT3 pathway. Am J Pathol 180: 351-364, 2012.

78. De la Iglesia N, Puram SV and Bonni A: STAT3 regulation of glioblastoma pathogenesis. Curr Mol Med 9: 580-590, 2009.

79. Kondo J, Endo H, Okuyama H, Ishikawa O, Lishi H, Tsujii M, Ohue $\mathrm{M}$ and Inoue $\mathrm{M}$ : Retaining cell-cell contact enables preparation and culture of spheroids composed of pure primary cancer cells from colorectal cancer. Proc Natl Acad Sci USA 108: 6235-6240, 2011. 